\title{
Lluvia de semillas y emergencia de plántulas de Fagus grandifolia subsp. mexicana en La Mojonera, Hidalgo, México
}

\author{
Seed rain and seedling emergence of Fagus grandifolia subsp. mexicana at La Mojonera, \\ Hidalgo, Mexico
}

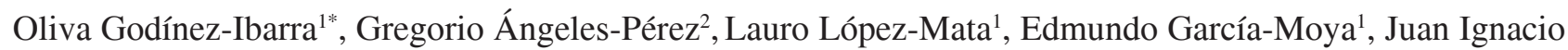
Valdez-Hernández ${ }^{2}$, Héctor De los Santos-Posadas² y Antonio Trinidad-Santos ${ }^{3}$

${ }^{1}$ Postgrado en Botánica, Colegio de Postgraduados. Montecillo 56230, Texcoco, Estado de México, México.

${ }^{2}$ Postgrado Forestal, Colegio de Postgraduados. Montecillo 56230, Texcoco, Estado de México, México.

${ }^{3}$ Postgrado en Edafología, Colegio de Postgraduados. Montecillo 56230, Texcoco, Estado de México, México.

*Correspondencia: goliva@colpos.mx

Resumen. Fagus grandifolia subsp. mexicana es una especie restringida a pequeñas poblaciones y sujeta a fuerte presión antropogénica. Con el objetivo de obtener información relevante que permita proponer alternativas de conservación, se analizó la producción de semillas y la demografía de plántulas de primer año en la Mojonera, Hidalgo. Se estableció una parcela de observación de $4800 \mathrm{~m}^{2}$ dividida en cuadrantes de 10 X $10 \mathrm{~m}$. Se utilizaron trampas de $0.5 \mathrm{~m}^{2}$ para estimar la producción de semillas, así como subparcelas de $1 \mathrm{~m}^{2}$ para registrar la emergencia y supervivencia de plántulas. La producción fue de 521667 semillas ha-1 $^{-1}$, de las que sólo el 24.44\% estaban llenas; el 46.01\% vanas, y el 29.55\% dañadas. La densidad de plántulas emergidas varió de 1 a 33 plántulas por $\mathrm{m}^{2}$. El porcentaje de supervivencia de plántulas de primer año fue de $2.8 \%$ después de 10 meses de observación, siendo las de mayor supervivencia las que emergieron durante las primeras fechas. El 34.44\% de las plántulas murieron por herbivoría, el $24.07 \%$ por damping-off y $23.65 \%$ por causa desconocida. La especie presenta el patrón general de supervivencia de especies arbóreas con alta mortalidad durante el primer año de vida.

Palabras clave: año semillero, demografía de plántulas, supervivencia de plántulas, causas de mortalidad.

Abstract. Fagus grandifolia subsp. mexicana is a species restricted to small populations under high anthropogenic pressure. With the aim to attain information to propose conservation strategies of this species, the seed rain and demography of current-year seedlings were analyzed at La Mojonera, Hidalgo, Mexico. A $4800 \mathrm{~m}^{2}$ plot divided into 10 X $10 \mathrm{~m}$ quadrants was established. The seed rain, seedling emergence, and survival were analyzed using seed traps of $0.5 \mathrm{~m}^{2}$ and adjacent $1 \mathrm{~m}^{2}$ sub-plots. The total seed rain was 521667 seeds ha $^{-1}$. A high proportion of seeds were unsound (46.01\%), followed by damaged seeds $\left(29.5 \%\right.$ ) and only $24.44 \%$ were sound. Emerged seedlings fluctuated from 1 to 33 seedlings $\mathrm{m}^{2}$. After 10 months, $2.8 \%$ of emerged seedlings were alive. Seedlings that emerged first showed higher survival probability. Important mortality causes were herbivory (34.44\%), damping-off (24.07\%) and unknown causes (23.65\%). This species presents the general pattern of tree species in seedlings survival, showing a high mortality rate during the first year.

Key words: mast year, seedling demography, seedling survival, mortality causes.

\section{Introducción}

La repoblación natural de especies arbóreas, se identifica como el paso de las poblaciones a través de sucesivos filtros ambientales que selectivamente eliminan

Recibido: 31 enero 2006; aceptado: 01 septiembre 2006 algunos individuos, mientras que otros pasan al siguiente estadio de desarrollo (Harper, 1977). En general, los cambios más drásticos en las poblaciones ocurren en los estadios de semilla y plántula (Brokaw, 1987; Harcombe, 1987; Lieberman y Lieberman, 1987; Swaine et al., 1987).

En el estadio de plántula las especies enfrentan las mayores presiones de selección, lo que resulta en elevadas 
tasas de mortalidad, característica común de la demografía de plántulas de especies arbóreas (Collins y Good, 1987; Shibata y Nakashizuka, 1995). Por esta razón, el tipo de repoblación de una especie, es del mayor interés al estudiar su dinámica poblacional (Álvarez-Buylla y MartínezRamos, 1990), debido a que los procesos que ocurren en esta etapa pueden ayudar a explicar varios aspectos de la población en el estadio adulto.

En las especies que presentan una producción sincrónica de semillas en diferentes años, llamados años semilleros, el estadio de semilla se convierte también en uno de los más importantes. Janzen $(1971,1978)$ argumentó que el año semillero es una respuesta evolutiva a la depredación, ya que una densidad alta de semillas saciará a los depredadores, permitirá que las semillas que no son consumidas puedan llegar a convertirse en individuos reproductivos y de esta forma se mantenga la población. El intervalo entre un año semillero y otro representa el trueque entre la necesidad de reducir la depredación y de maximizar la oportunidad de reproducción (Taylor y Aarsen, 1989).

Las limitaciones básicas en la incorporación de nuevos individuos son la disponibilidad de semillas o propágulos y la disponibilidad de sitios adecuados, seguros para su establecimiento (Ward, 1961; Glitzenstein et al.,1986; Erickson y Ehrlen, 1992). Varios estudios han demostrado que la disponibilidad de luz, microtopografía, tipo de sustrato, profundidad del mantillo, y cobertura de la vegetación rasante, son factores importantes que afectan la supervivencia de las plántulas (Facelli y Pickett, 1991; Jones et al., 1994, Shibata y Nakashizuka, 1995; Seiwa, 1998; Ángeles-Pérez y Sakimoto, 1999).

La identificación de las causas probables de mortalidad y los factores que rigen la demografía de plántulas de primer año son de especial importancia para explicar los mecanismos que permiten el mantenimiento de la reserva de plántulas en el piso forestal de especies con producción de semillas en períodos relativamente espaciados.

Los árboles de Fagus grandifolia Ehrh. subsp. mexicana (Martínez) E. Murray (Fagaceae) presentan en México una producción abundante de semillas cada 4 a 7 años (Pérez-Rodríguez, 1999) en la población ubicada en La Mojonera, Hidalgo. Esta especie, tiene una distribución natural muy restringida y confinada a 10 poblaciones en el bosque mesófilo de montaña de la Sierra Madre Oriental (Williams-Linera et al., 2003; Rowden et al., 2004).

La distribución restringida y el reducido tamaño de las poblaciones de F. grandifolia subsp. mexicana en bosques mesófilos de la Sierra Madre Oriental de México, la coloca en algún estado de riesgo e incluso la hace susceptible a la extinción. Por tanto, es necesario el estudio de la ecología de sus poblaciones relictas, porque ello proporcionará información que podrá utilizarse para proponer mecanismos viables para su conservación (Williams-Linera et al.,
2003).

El objetivo de esta investigación es el estudio de la demografía de una cohorte de plántulas de F. grandifolia subsp. mexicana de primer año de vida en una parcela de observación permanente en La Mojonera, Zacualtipán, Hidalgo. Debido a que ésta es una especie que presenta la característica de "año semillero" se hizo un análisis de la producción de semillas de la población en el lugar.

\section{Materiales y métodos}

Área de estudio. El área de estudio se localiza en el ejido La Mojonera del municipio de Zacualtipán (20³9'41" N, 98`39'17' O), en el estado de Hidalgo. La región se encuentra en la Provincia fisiográfica de la Sierra Madre Oriental y sub-provincia Sierra de Zacualtipán, y se caracteriza por presentar un relieve accidentado con pendientes mayores a 45\% (Pérez-Rodríguez, 1999). El material litológico predominante son rocas volcánicas terciarias y cuaternarias. Los suelos predominantes son Andisol vítrico y húmico, según la clasificación de la FAOUNESCO. La altitud varía de 1900 a $2200 \mathrm{~m}$. El clima es C(fm) templado húmedo con lluvias todo el año (García, 1988). La temperatura media anual es de $12.7^{\circ} \mathrm{C}$, con una máxima extrema promedio de $38^{\circ} \mathrm{C}$ y mínima extrema de $-10^{\circ} \mathrm{C}$. La precipitación anual es de $2047 \mathrm{~mm}$. Las neblinas se presentan durante todo el año y en promedioel número de días nublados es de 174 (Servicio Meteorológico Nacional, 1975).

En la parte nororiental de Zacualtipán se encuentra una población de Fagus grandifolia subsp. mexicana con superficie de 45 ha. Esta población es la de mayor extensión y mejor conservada en México (Ehnis,1981; Peters, 1995; Williams-Linera et al., 2003). El estrato superior se encuentra dominado por F. grandifolia subsp. mexicana, en el que se asocian en menor grado especies como Magnolia schiedeana Schlecht., Clethra macrophylla M. Martens et Galeotti y varias especies de Quercus. El sotobosque es muy escaso debido a las limitadas condiciones de luz. El estrato intermedio lo constituyen especies como Eugenia capuli (Schltdl. et Cham) Hook. et Arn., Ocotea klotzschiana (Nees) Hemsl. y especies de Cestrum, y el estrato inferior está dominado por los géneros Miconia, Deppea y Elaphoglossum.

En este bosque se estableció una parcela de observación de forma rectangular con dimensiones de 80 × 60 m (4800 $\mathrm{m}^{2}$ ). La parcela se dividió en una retícula de 48 cuadros de $10 \mathrm{~m}$ x $10 \mathrm{~m}$. Los cuadros se señalaron con estacas de madera en sus vértices. Para la caracterización estructural de la parcela, dentro de cada cuadro se numeraron todos los individuos arbóreos con diámetro a la altura del pecho 
(DAP) $\geq 1.0 \mathrm{~cm}$, se identificaron y se localizaron por sus coordenadas cartesianas (x, y). Para el caso de F. grandifolia subsp. mexicana, todos los individuos con altura $(\mathrm{h}) \geq 0.6$ $\mathrm{m}$ fueron numerados, registrados y localizados.

Lluvia de semillas. La estimación de la producción de semillas se realizó mediante el estudio de la lluvia de semillas de la especie. Se utilizaron 48 trampas circulares de $0.5 \mathrm{~m}^{2}$ colocadas en el centro de cada uno de los cuadros de $10 \mathrm{~m}$ x $10 \mathrm{~m}$, a $1.0 \mathrm{~m}$ de altura del piso forestal. La instalación de las trampas se hizo a principios del mes de mayo, antes del periodo de liberación de las semillas. Las semillas colectadas en las trampas se recogieron mensualmente y se caracterizaron como llenas, vanas o dañadas. Para discriminar las semillas llenas de las vanas, algunas se analizaron mediante disección. Las dañadas fueron aquellas que presentaron indicios de haber sido consumidas por insectos, tales como un orificio visible o muestras de excremento.

Emergencia y supervivencia de plántulas. La demografía de las plántulas de primer año, se estudió a través de su emergencia y supervivencia. Se establecieron subparcelas de $1 \mathrm{~m}^{2}$ al lado de la trampa de semillas para evaluar la emergencia de plántulas. Dentro de cada subparcela se registraron las plántulas emergidas, las cuales se marcaron para dar seguimiento a su supervivencia. En cada censo semanal se registraron las nuevas plántulas emergidas y se determinaron las posibles causas de mortalidad.

Las posibles causas de mortalidad se clasificaron en 5 categorías (Shibata y Nakashizuka, 1995; Ángeles-Pérez y Sakimoto, 1999; Moles y Westoby, 2004): 1) herbivoría, para las plántulas muertas con cotiledones o hipocótilo consumidos; 2) damping-off (ahogamiento), para las plántulas muertas con indicios de tallo podrido a nivel de suelo causado por hongos; 3) sequía, para plántulas presentes en el suelo pero con los cotiledones secos; 4) daño físico, para plántulas que murieron por micro derrumbes (exposición del sistema radical), rodamiento de piedras o disturbios en el suelo; y 5) desconocida, para plántulas que desaparecieron de la subparcela.

Análisis estadístico. La lluvia de semillas se analizó bajo el enfoque de muestreo simple aleatorio y los valores estimados de producción de semillas por hectárea que se ofrecen usan los estimadores clásicos de este tipo de muestreo (Cochran, 1980). De esta forma se calculó el número de semillas por hectárea en el bosque de $F$. grandifolia subsp. mexicana.

Para la supervivencia de las plántulas tanto a nivel de subparcela como de plántula se planteó el uso de un modelo lineal generalizado (MLGEN). Dado que los datos de supervivencia no se distribuyen de manera normal, se utilizó la distribución binomial vinculada directamente con un módulo lineal a una función log-log (Agresti, 2002). El ajuste se realizó con el procedimiento GENMOD de SAS (SAS, 1997).

El ajuste de la supervivencia total por parcela fue descrito mediante una estructura de vínculo log-log complementaria. El módulo lineal utilizado parte de la forma básica $\boldsymbol{n}=\beta_{0}+\beta_{1} \boldsymbol{t}$. Donde $\boldsymbol{n}$ es el módulo lineal de la función log-log complementaria, $\beta_{i}$ son los parámetros del módulo y $\boldsymbol{t}$ es el tiempo en días en el que se da el cambio de la proporción de plantas vivas y muertas.

De esta forma la mortalidad es una función del tiempo en días a partir de la emergencia. Evidentemente la supervivencia dada por $\beta_{1}$ depende del momento en que hayan emergido las plántulas y la tasa de mortalidad varía con la fecha de emergencia. Para considerar ambas hipótesis, el módulo lineal se amplió a la siguiente expresión:

$$
\begin{gathered}
\boldsymbol{n}=\beta_{0}+\beta_{01} I_{F 1}+\beta_{02} I_{F 2}+\beta_{04} I_{F 4}+\left(\beta_{1}+\beta_{12} I_{F 2}+\right. \\
\left.\beta_{13} I_{F 3}+\beta_{14} I_{F 4}\right) \times \boldsymbol{t}
\end{gathered}
$$

donde: $I_{F k}$ es la variable indicadora por cada una de las $k$ fechas de emergencia definidas como:

$\begin{array}{ll}\text { 16-Sep } & \text { Fecha } 1 \\ \text { 24-Sep } & \text { Fecha } 2 \\ \text { 02-Oct } & \text { Fecha } 3 \\ \text { 08-Oct } & \text { Fecha } 4\end{array}$

El valor de referencia del modelo fue la fecha 5 que la componen todas las observaciones tomadas después del 15 de octubre (fechas de emergencia del 15, 22 y 28 de octubre), ya que las 2 últimas fechas tienen muy pocas observaciones para poder lograr que los parámetros que las componen generen valores estimados para los parámetros del modelo definidos en 7 clases.

\section{Resultados}

El bosque de F. grandifolia subsp. mexicana en La Mojonera, Zacualtipán, está compuesto principalmente por 8 especies arbóreas y otras de menor importancia estructural (Cuadro 1). Este bosque está claramente dominado por F. grandifolia subsp. mexicana con un valor de importancia relativo (VIR) de 63.57 y como codominantes se encuentran Nectandra salicifolia con un VIR de 9.21 y Quercus affinis con 8.63 de VIR. La densidad de árboles por hectárea es de 2119 que alcanzan un total de $43.34 \mathrm{~m}^{2}$ de área basal. 
Cuadro 1. Composición de especies del bosque de Fagus grandifolia subsp. mexicana en La Mojonera, Zacualtipán, Hidalgo. Las especies están ordenadas descendentemente de acuerdo con su valor de importancia relativo (VIR).

\begin{tabular}{lccccc}
\hline Especie & $\begin{array}{c}\text { Densidad } \\
\text { (ind/ha) }\end{array}$ & $\begin{array}{c}\text { Densidad } \\
(\%)\end{array}$ & $\begin{array}{c}\text { Área basal } \\
\left(\mathrm{m}^{2} / \mathrm{ha}\right)\end{array}$ & $\begin{array}{c}\text { Área basal } \\
(\%)\end{array}$ & VIR $^{*}$ \\
\hline Fagus grandifolia subsp. mexicana & 954 & 45.03 & 35.58 & 82.10 & 63.57 \\
Nectandra salicifolia & 335 & 15.83 & 1.12 & 2.59 & 9.21 \\
Quercus affinis & 148 & 6.98 & 4.45 & 10.28 & 8.63 \\
Symplocos limoncillo & 181 & 8.55 & 0.20 & 0.45 & 4.50 \\
Magnolia schiedeana & 144 & 6.78 & 0.68 & 1.58 & 4.18 \\
Clethra macrophylla & 125 & 5.90 & 0.70 & 1.63 & 3.76 \\
Nectandra heydeana & 111 & 5.21 & 0.14 & 0.33 & 2.77 \\
Ilex discolor & 65 & 3.05 & 0.05 & 0.11 & 1.58 \\
Befaria aestuans & 4 & 0.20 & 0.25 & 0.58 & 0.39 \\
Cleyera integrifolia & 13 & 0.59 & 0.06 & 0.15 & 0.37 \\
Prunus serotina subsp. capuli & 8 & 0.39 & 0.01 & 0.02 & 0.21 \\
Quercus sp. & 4 & 0.20 & 0.02 & 0.05 & 0.12 \\
Ternstroemia sylvatica & 4 & 0.20 & 0.01 & 0.01 & 0.10 \\
Otras especies & 23 & 1.08 & 0.06 & 0.13 & 0.61 \\
Total & 2119 & & 43.34 & & \\
\hline
\end{tabular}

*el VIR es un promedio de los porcentajes de densidad y área basal relativas.

Lluvia de semillas. La población de F. grandifolia subsp. mexicana en La Mojonera presentó una densidad variable en la lluvia de semillas, tanto en el número total por trampa como en el número de semillas llenas por trampa que se registraron durante todo el estudio (Figs. 1A y 1B, respectivamente). En una trampa no hubo presencia de semillas, mientras que en 15, las semillas fueron vanas o dañadas. La presencia de semillas llenas en las trampas también fue variable en la parcela. La producción de semillas fue de 521667 por ha, el mayor porcentaje, $46.01 \%$, corresponde a semillas vanas, seguidas por las dañadas con el $29.55 \%$ y las llenas con el $24.44 \%$ (Cuadro 2).

La dispersión de las semillas comenzó en junio; sin embargo, la mayoría de ellas fueron vanas o dañadas. En septiembre se dio la mayor producción de semillas de $F$. grandifolia subsp. mexicana, seguida por la de agosto y julio. En enero se obtuvo el último registro de lluvia de semillas en las trampas. La presencia de semillas llenas se registró a partir de agosto, prolongándose hasta octubre, y fue también en septiembre cuando cayó la mayor cantidad de éstas (Fig. 2).

Emergencia y supervivencia de plántulas. Se observó gran variación en el número de semillas emergidas en toda la parcela, concentrándose de manera preferencial en la parte baja (Fig. 1C). La densidad de plántulas emergidas por metro cuadrado osciló de una a 33 plántulas; las subparcelas con menos de 10 plántulas fueron las de mayor frecuencia. La emergencia de las plántulas comenzó el 16 de septiembre y terminó el 28 de octubre. La máxima emergencia se presentó en el período comprendido entre el 24 de septiembre y el 15 de octubre (Fig. 3). Del total de 248 plántulas emergidas en los $48 \mathrm{~m}^{2}$, hasta el 30 de junio sólo sobrevivían 7 (Fig. 1D, Fig. 3).

La mortalidad de plántulas se presentó a partir de la primera semana de octubre; fue máxima en las subsecuentes 3 fechas de censo (Fig. 5). La supervivencia de las plántulas de primer año presentó valores más altos en el mes de octubre. Para los meses siguientes de noviembre a junio, la supervivencia de las plántulas disminuyó de manera constante. Para finales de junio, la supervivencia fue muy baja con sólo el 2.8\% del total de plántulas emergidas (Fig. $3)$. En general, las plántulas que emergieron durante las primeras fechas de registro, presentaron mayor tasa de supervivencia; las 7 plántulas vivas hasta el 30 de junio corresponden a las emergidas el 24 de septiembre y el 2 y 8 de octubre (Fig. 4).

El análisis de supervivencia mostró que la fecha de emergencia es determinante en la supervivencia de las 

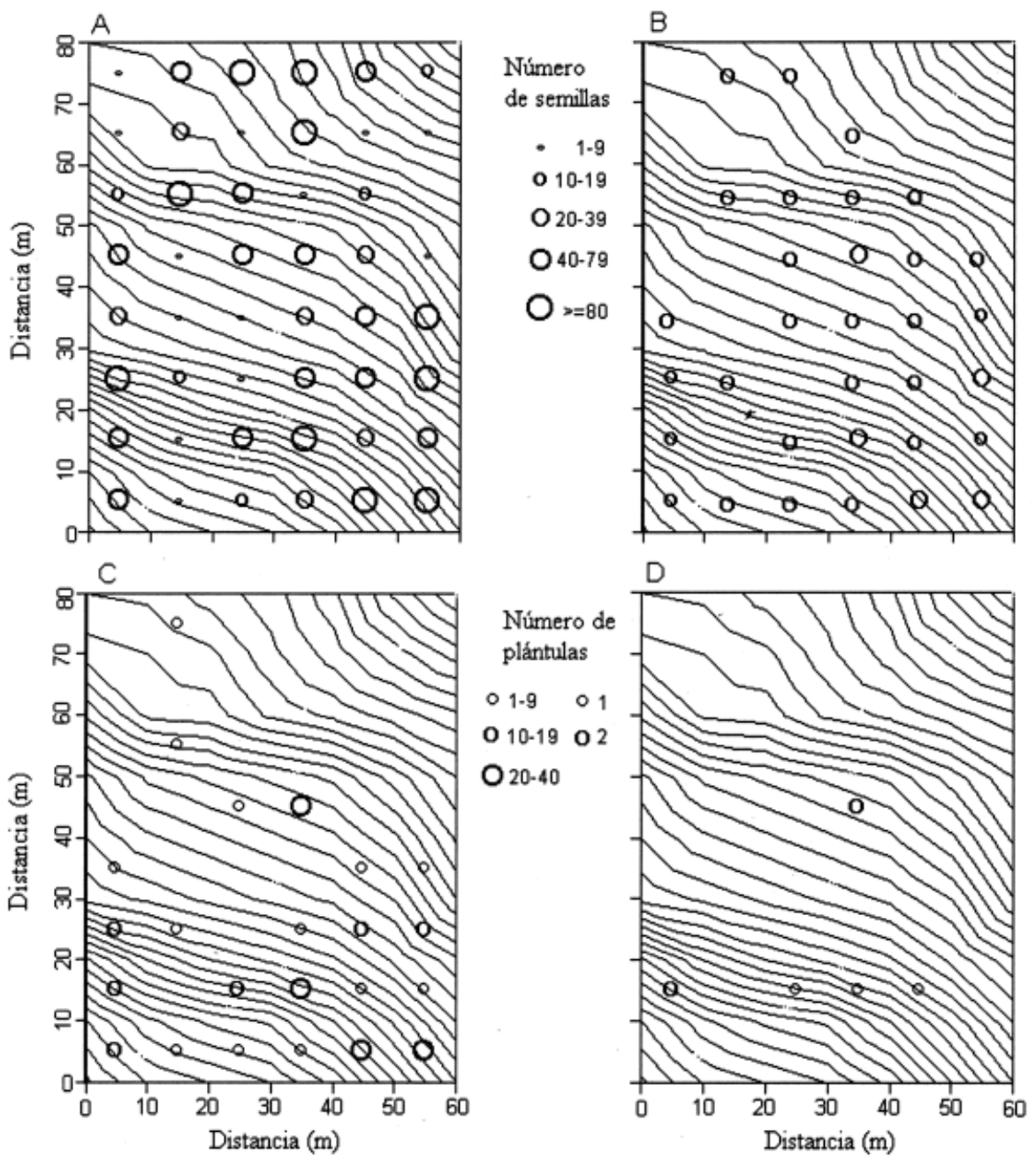

Figura 1. Fagus grandifolia subsp. mexicana. Número de semillas al final del estudio. A, totales (llenas, dañadas y vanas)y B, sólo semillas llenas. Número de plántulas al 30 de junio. C, emergidas y D) supervivientes El tamaño del círculo indica la densidad de semillas en cada trampa de $0.5 \mathrm{~m}^{2}$ y plántulas en cada subparcela de $1 \mathrm{~m}^{2}$. Las líneas indican curvas de nivel cada $2 \mathrm{~m}$, y disminuyen en altitud de derecha a izquierda y de arriba hacia abajo.

plántulas (Cuadro 3). Así, el hecho de que los parámetros $\beta_{01}$ y $\beta_{02}$ sean positivos y estadísticamente diferentes significa mayores probabilidades iniciales de supervivencia para las plántulas nacidas antes de octubre. No así la fecha 4 que es la que tiene para los datos las condiciones menos favorables de supervivencia inicial. Las plántulas que emergieron el 16 y 24 de septiembre tuvieron una mayor probabilidad de sobrevivir que aquellas que emergieron en fechas posteriores (Fig. 4).

La fecha 2 tiene la tasa unitaria de mortalidad menor $\beta_{1}+$ $\beta_{12}=-0.198$, seguida de las fechas 3 y 4 cuya mortalidad aproximada es -0.3041 y -0.3308 , respectivamente. La tasa de mortalidad del módulo lineal en cualquiera de las otras fechas es muy acelerada incluyendo la fecha 1 (-
Cuadro 2. Producción de semillas por hectárea de Fagus grandifolia subsp. mexicana al final del estudio para cada condición de la semilla, se incluyen los intervalos de confianza al $95 \%$ inferior y superior y el porcentaje, ambos con referencia a la densidad total.

\begin{tabular}{lcccc}
\hline $\begin{array}{c}\text { Condición } \\
\text { de la } \\
\text { semilla }\end{array}$ & $\begin{array}{c}\text { Densidad } \\
\text { (No. /ha) }\end{array}$ & $\begin{array}{c}\text { Intervalos de confianza } \\
\text { al 95\% }\end{array}$ & $\%$ \\
\hline Llena & 127,500 & 75879.11 & 179120.89 & 24.44 \\
Vana & 240,000 & 125690.48 & 354309.52 & 46.01 \\
Dañada & 154,167 & 101669.34 & 206663.99 & 29.55 \\
Total & 521,667 & 348024.69 & 695308.64 & \\
\hline
\end{tabular}




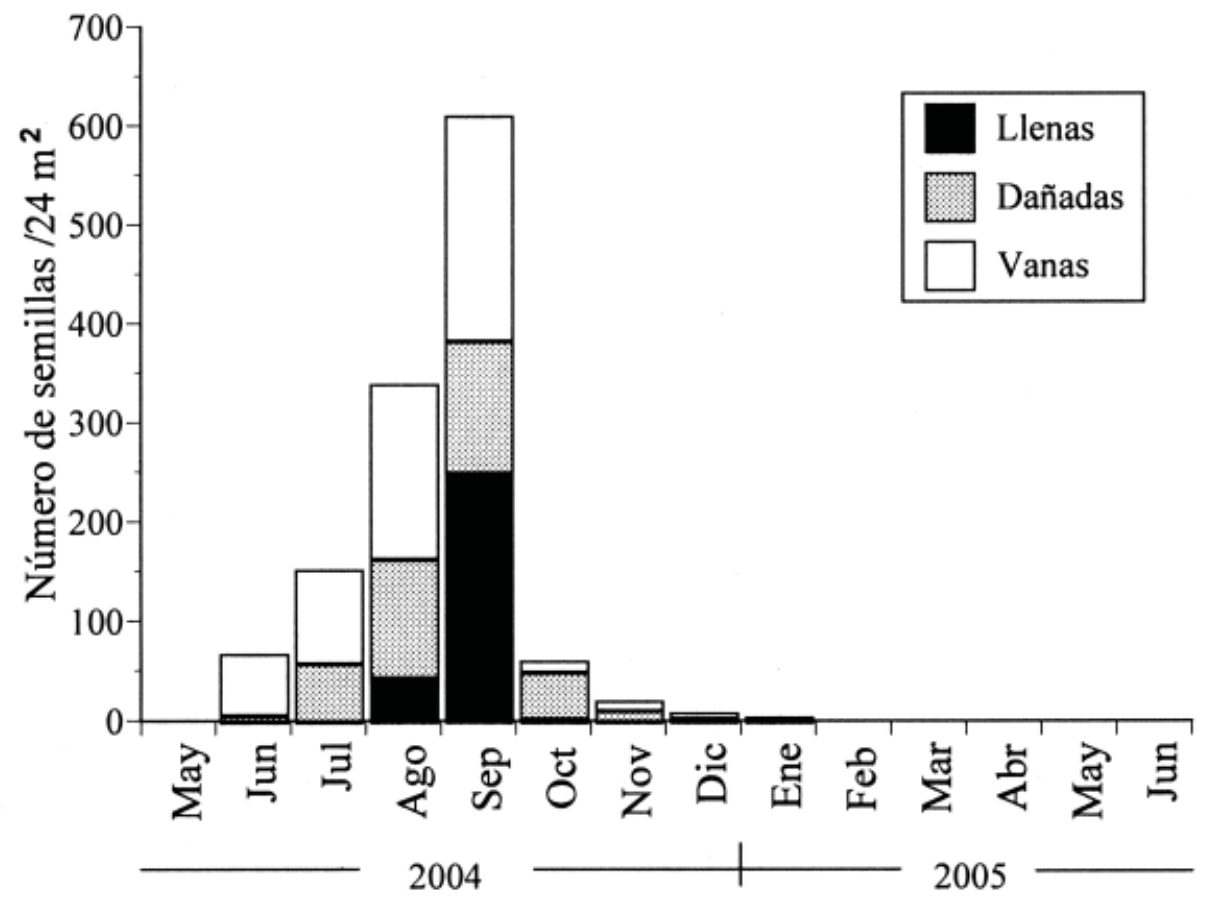

Figura 2. Cantidad y condición de las semillas de Fagus grandifolia subsp. mexicana dispersadas en 48 trampas de $0.5 \mathrm{~m}^{2}$.

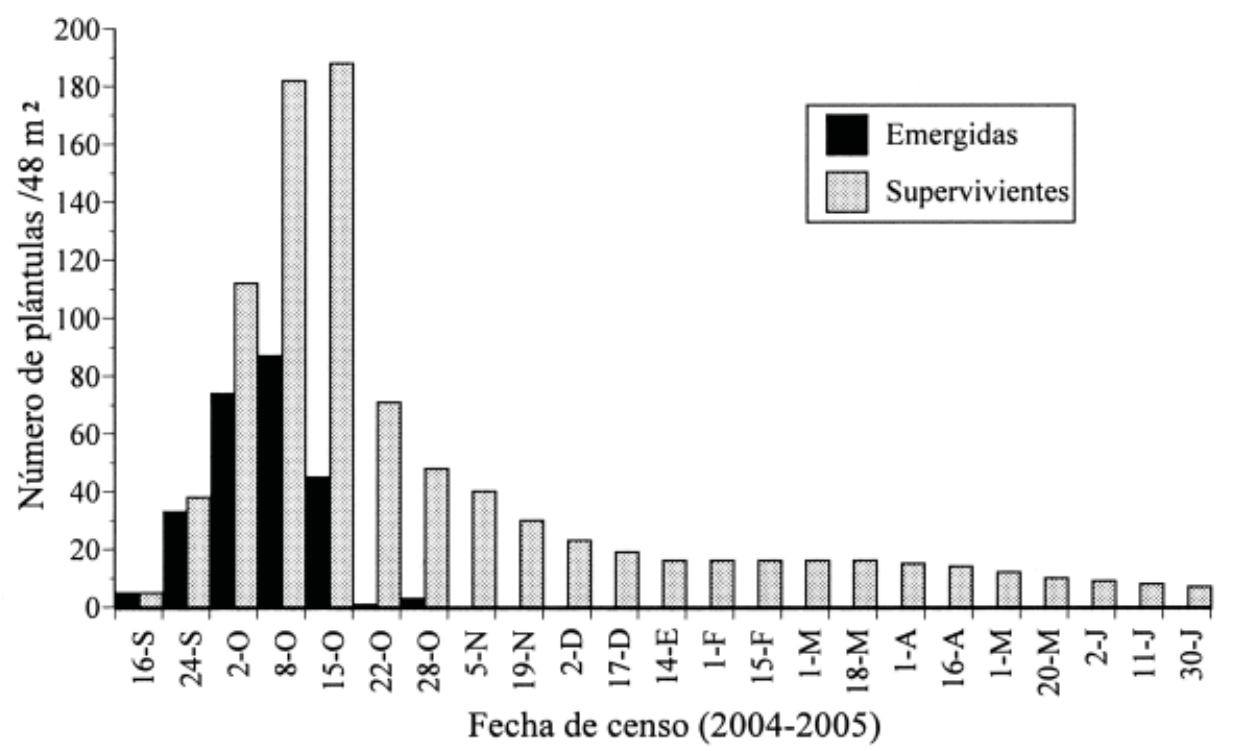

Figura 3. Emergencia y supervivencia de plántulas de primer año de Fagus grandifolia subsp. mexicana por fecha de censo. 


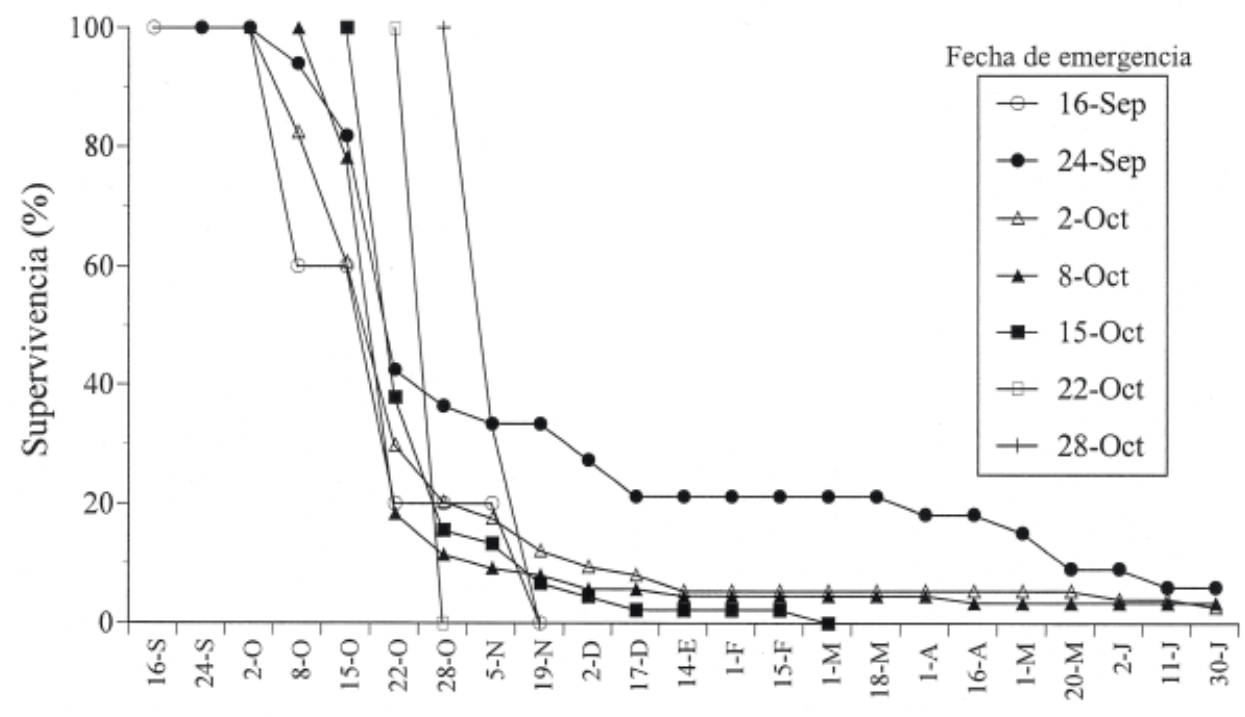

Fecha de censo (2004-2005)

Figura 4. Curvas de supervivencia de plántulas de primer año de Fagus grandifolia subsp. mexicana por fecha de emergencia.

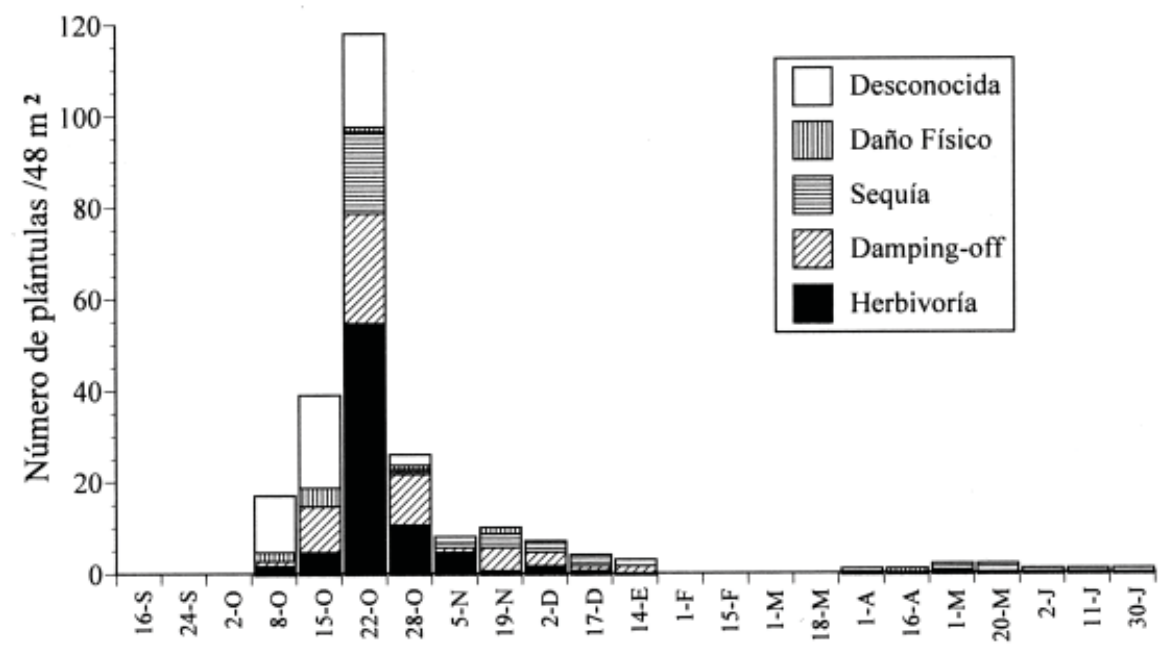

Fecha de censo (2004-2005)

Figura 5. Probables causas de mortalidad de las plántulas de primer año de Fagus grandifolia subsp. mexicana, en cada fecha de censo. 
Cuadro 3. Bondad de ajuste y parámetros estimados para la supervivencia por fecha de emergencia de las plántulas de primer año de Fagus grandifolia subsp. mexicana.

\begin{tabular}{llcc}
\hline & \multicolumn{3}{c}{ Criterios de bondad de ajuste } \\
\hline Criterio de ajuste & $G L$ & Valor & Valor/GL \\
& & & \\
Desvianza & 75 & 434.7653 & 5.7969 \\
Ji-cuadrada de Pearson & 75 & 493.1563 & 6.5754 \\
Log-máxima verosimilitud & -1478.0717 & & \\
\hline
\end{tabular}

\begin{tabular}{clllllll}
\hline \multicolumn{7}{c}{ Parámetros estimados } \\
\hline Parámetro & GL & $\begin{array}{l}\text { Valor } \\
\text { estimado }\end{array}$ & $\begin{array}{l}\text { Error } \\
\text { estándar }\end{array}$ & $\begin{array}{l}\text { Intervalo de 95\% del } \\
\text { valor estimado }\end{array}$ & $\begin{array}{l}\text { Ji-cuadrada } \\
\text { calculada }\end{array}$ & $\begin{array}{l}\text { Valores de } \\
\text { rechazo }\end{array}$ \\
\hline$\beta_{0}$ & 1 & 0.4906 & 0.0810 & 0.3310 & 0.6487 & 36.68 & $<.0001$ \\
$\beta_{01}$ & 1 & 2.2652 & 0.3713 & 1.5409 & 2.9955 & 37.22 & $<.0001$ \\
$\beta_{02}$ & 1 & 0.3716 & 0.1423 & 0.0927 & 0.6509 & 6.82 & 0.0090 \\
$\beta_{04}$ & 1 & -0.3702 & 0.1270 & -0.6201 & -0.1220 & 8.49 & 0.0036 \\
$\beta_{1}$ & 1 & -0.7785 & 0.0632 & -0.9106 & -0.6623 & 151.71 & $<.0001$ \\
$\beta_{12}$ & 1 & 0.5805 & 0.0649 & 0.4605 & 0.7155 & 80.03 & $<.0001$ \\
$\beta_{13}$ & 1 & 0.4744 & 0.0603 & 0.3639 & 0.6008 & 61.99 & $<.0001$ \\
$\beta_{14}$ & 1 & 0.4477 & 0.0667 & 0.3236 & 0.5857 & 45.06 & $<.0001$ \\
\hline
\end{tabular}

0.7785). Tomando en cuenta que son específicos del año y temporada en que se realizó el estudio, estos resultados sugieren que la ventana en la cual las condiciones óptimas de supervivencia de las plántulas de la repoblación se da sólo durante un par de semanas al año en el mes de septiembre, cuando se presentó la mayor liberación de semillas (Fig. 2).

La revisión periódica de la supervivencia y mortalidad de las plántulas permitió registrar las posibles causas de mortalidad (Fig. 5). Las 5 causas de mortalidad estuvieron presentes en las fechas de mayor mortalidad; sin embargo, se presentó cierta variación estacional. Del 15 al 22 de octubre, cuando se observó la máxima mortalidad, se presentaron las 5 causas de muerte, la más importante fue la herbivoría, seguida de damping-off. Para las fechas posteriores de censo, las causas más comunes de mortalidad fueron las mismas pero en proporciones diferentes. En los censos de abril a junio, la causa predominante fue la sequía. En general en este bosque, las causas probables de mortalidad de plántulas de primer año de F. grandifolia subsp. mexicana son herbivoría con $34.44 \%$, seguida del damping-off con $24.07 \%$, la desconocida con $23.65 \%$, la sequía con $13.69 \%$ y el daño físico con $4.15 \%$.

\section{Discusión}

La composición de especies arbóreas del bosque de $F$. grandifolia subsp. mexicana de La Mojonera, Zacualtipán, Hidalgo, concuerda con lo referido para este bosque y para otros de la misma región (Alcántara y Luna, 2001; Luna y Alcántara, 2004), un bosque con claro predominio estructural de Fagus con más del $45 \%$ de densidad y más de $80 \%$ de área basal, como se menciona para un bosque similar en una población en Acatlán, Veracruz (ÁlvarezAquino, 1997).

Producción de semillas. La producción de semillas de esta especie en 2004 fue variable en toda la parcela. En promedio, la densidad fue de 52.2 semillas $\mathrm{m}^{-2}$. No existen informes para la especie en otras poblaciones que permitan confrontar estos valores y determinar qué tan baja o alta es esta producción. Sin embargo, Houle (1992) consigna promedios de 13.30 semillas $\mathrm{m}^{-2}, 19.94$ semillas $\mathrm{m}^{-2} \mathrm{y}$ 23.27 semillas $\mathrm{m}^{-2}$ en la lluvia de semillas de F. grandifolia 
en Norte América, lo que sitúa al bosque de La Mojonera con una mayor producción. Mientras que para F. crenata se mencionan hasta 47.8 semillas $\mathrm{m}^{-2}$ (Tomita et al., 2002). Para F. sylvatica se registran años semilleros con producción baja, intermedia y alta, y ello determina no sólo la cantidad sino también en gran medida la calidad de las semillas (Nilsson, 1985).

El estadio de la semilla al momento de su liberación de las cápsulas es, sin duda, determinante en el potencial de germinación. En este estudio, el mayor porcentaje de las semillas que cayeron fueron vanas $(46.01 \%)$, en comparación con las semillas llenas (24.44\%) (Cuadro 2). Estas últimas tuvieron una capacidad germinativa de $75.4 \%$. Resultados semejantes se han encontrado para otras especies en el hemisferio norte. Más del $60 \%$ de las semillas de F. sylvatica que se dispersan corresponden a semillas vanas o depredadas por vertebrados e invertebrados (Nilsson, 1985). De F. crenata se ha informado que en años con baja producción de semillas, cerca del $90 \%$ las consumen vertebrados e invertebrados (Yasaka et al., 2003; Ida et al., 2004).

Emergencia y mortalidad de plántula. Los procesos que ocurren durante el estadio de plántulas tienen un gran impacto en la población de adultos de especies arbóreas (Clark y Clark, 1989), y en bosques naturales el piso forestal que provee un lecho para la emergencia de las plántulas y la subsiguiente supervivencia es heterogénea en el tiempo y el espacio (Ángeles-Pérez y Sakimoto, 1999). Esta heterogeneidad espacial explica en parte la densidad diferencial en la emergencia de las plántulas de F. grandifolia subsp. mexicana en este bosque. En condiciones controladas, las semillas provenientes del sitio de estudio presentaron un porcentaje de germinación del $75.4 \%$, mientras que semillas de la misma especie de las poblaciones de Acatlán, Veracruz, presentaron un porcentaje de germinación mayor al 80\% (Álvarez-Aquino y Williams-Linera, 2002).

La densidad de plántulas $\left(10.8 \pm 2.1\right.$ plántulas $\left.\mathrm{m}^{-2}\right)$ es baja comparada con la que se menciona para el bosque de la misma especie en Acatlán, Veracruz de 51.5 plántulas $\mathrm{m}^{-2}$ (Álvarez-Aquino y Williams-Linera, 2002); pero es una densidad alta al compararla con la de los bosques de F. grandifolia en Estados Unidos de América que presentan 2.4 plántulas $\mathrm{m}^{-2}$ (Wisconsin), 4.7 plántulas $\mathrm{m}^{-}$ 2 (Tennessee), 4.0 plántulas $\mathrm{m}^{-2}$ (Indiana), 3.2 plántulas $\mathrm{m}^{-2}$ (Ohio) y 0.6 plántulas $\mathrm{m}^{-2}$ (Michigan) (Ward, 1961). Mientras que para F. sylvatica en Suecia la densidad fue de 0.2 plántulas $\mathrm{m}^{-2}$ (Nilsson, 1985), y la de F. crenata en Japón fue de 1.0 plántulas $\mathrm{m}^{-2}$ (Hara, 1987) y 2.9 plántulas $\mathrm{m}^{-2}$ (Akashi, 1997).

La presencia del año semillero no garantiza un alto porcentaje de establecimiento de plántulas en el piso forestal (Álvarez-Aquino y Williams-Linera, 2002), debido a que normalmente, después de dispersadas, una alta proporción de las semillas es atacada por patógenos o consumida por vertebrados, aunque existen evidencias que la presencia del año semillero es una respuesta evolutiva para el escape al ataque de depredadores (Nilsson y Wästljung, 1987). En este estudio, de la producción total de semillas, únicamente emergieron plántulas del 9.9\%. Sin embargo, este porcentaje es mayor al registrado en Suecia, ya que después del año semillero la densidad de plántulas de F. sylvatica fue de $0.04 \%$ de la producción estimada de semillas (Nilsson, 1985). Para las poblaciones de Fagus, el paso de semilla a plántulas es un importante cuello de botella. Después, el estadio de plántula es también muy sensible, por lo que el reclutamiento al siguiente estadio (juveniles) de vida es casi nulo.

Las plántulas de F. grandifolia subsp. mexicana en La Mojonera que emergieron primero, mostraron tendencia a mayores tasas de supervivencia (aunque nula), lo que permite establecer que la fecha de emergencia es importante en la supervivencia de las mismas. Resultados semejantes se han encontrado en Abies firma (Ángeles-Pérez y Sakimoto, 1999), Carpinus spp. (Shibata y Nakashizuka, 1995), Acer rubrum (Jones et al., 1997), Acer mono (Seiwa, 1998), F. sylvatica (Szwagrzyk et al., 2001), entre otras especies. Las hipótesis que intentan explicar este fenómeno se han clasificado en 2 categorías: efectos genéticos y maternos y efectos ambientales (Jones et al., 1997). La primera propone que las semillas más grandes y, por tanto, más vigorosas, o bien, las que poseen genotipos mejor adaptados tienden a germinar primero, lo que implica que el tiempo de emergencia de las plántulas está correlacionado con el tamaño de la semilla. La segunda, propone que las semillas que germinan primero toman ventaja de ciertos factores ambientales que están disponibles en forma temporal al momento de la germinación, tales como luz y disponibilidad de agua o nutrimentos (Jones y Sharitz, 1989).

Una diferencia importante de las especies antes mencionadas respecto a F. grandifolia subsp. mexicana es que sus semillas acumuladas en el piso forestal pasan un período de bajas temperaturas durante el invierno y la emergencia ocurre en la siguiente estación de crecimiento. Aquellas plántulas que emergen antes de que las especies en el dosel expandan sus hojas tienen una ventaja comparativa que les permite un uso eficiente de la luz disponible en el sotobosque. En contraste, las semillas de F. grandifolia subsp. mexicana germinan poco después de dispersarse, si la disponibilidad de humedad es adecuada. En el bosque mesófilo de montaña hidalguense, a menudo, el agua no es el factor limitante para la emergencia de las plántulas; más bien, con frecuencia, se convierte en un factor limitante 
si se presenta en exceso, ya que propicia el ataque de diversas especies de hongos que ocasionan pudriciones en el suelo. De hecho, el damping-off fue una de las causas de mortalidad que estuvo presente durante los primeros meses después de la emergencia de las plántulas (Fig. 5).

La supervivencia de las plántulas de F. grandifolia subsp. mexicana fue muy baja (2.8\% en 10 meses), comparada con la consignada en Acatlán para la misma especie (17\% en 16 meses; Álvarez-Aquino y WilliamsLinera, 2002). En otras especies también se observó mayor porcentaje de supervivencia, como en Abies firma (40.85\% en 9 meses; Ángeles-Pérez y Sakimoto, 1999), Carpinus spp. (< $10 \%$ en 7 meses; Shibata y Nakashizuka, 1995) y Acer mono (de 55 a $68 \%$ en 7 meses; Seiwa, 1998). La elevada tasa de mortalidad en plántulas es característica de muchas especies de árboles y ello puede producir la selección natural más intensa en todo el ciclo de vida de la planta y afectar fuertemente los patrones de distribución de los adultos (Clark y Clark, 1989).

Las plántulas de F. grandifolia subsp. mexicana murieron principalmente a causa de la herbivoría. No se hizo un análisis específico sobre los depredadores, pero se observaron ortópteros (Fig. 5). El predominio de las causas de mortalidad de plántulas en las diferentes especies es diferencial, en F. crenata las principales son la depredación y el damping-off (Abe et al.,2001), para Abies firma (Ángeles-Pérez y Sakimoto, 1999), al igual que en plántulas de F. grandifolia subsp. mexicana, la herbivoría, el damping-off y la sequía predominaron en un principio, considerando que esta última causa es muy específica tanto de la especie como del sitio; y estas mismas son las que se consignan como las principales causas de mortalidad en plántulas de otras especies (Moles y Westoby, 2004).

La menos importante entre las causas de mortalidad para las plántulas de F. grandifolia subsp. mexicana es el daño físico ocasionado por caída de ramas y hojarasca, rocas rodantes, derrumbes y el pisoteo por vertebrados del sotobosque, entre otros. Todos estos agentes potencialmente mortales son importantes en el bosque de Fagus, donde las variaciones topográficas, de luminosidad y de humedad son muy altas. Cualesquiera que sean las causas de mortalidad de plántulas de especies arbóreas está documentado que es muy elevada durante el primer año de vida, pero que disminuye en función de las condiciones ambientales prevalecientes (Berkowitz et al., 1995) y de las características propias de las especies.

Para F. grandifolia subsp. mexicana, después de 10 meses de observación, se registró una supervivencia de sólo $2.8 \%$ de las plántulas que emergieron, tasa que es relativamente baja comparada con la de otras áreas de su distribución. Si con esta incorporación de plántulas se asegura o no la permanencia de la especie, es una pregunta que necesita contestarse por medio de la observación de la supervivencia de las plántulas que aún están vivas, así como de las siguientes generaciones de plántulas, producto de años semilleros en el futuro. Por otro lado, es importante analizar la dinámica en el reclutamiento de individuos hacia subsecuentes estadios de desarrollo.

\section{Agradecimientos}

Al proyecto SEP-CONACyT 44872 "Dinámica y productividad del bosque mesófilo de montaña en la sierra hidalguense" con el cual se financió este estudio.

\section{Literatura citada}

Abe, M., H. Miguchi y T. Nakashizuka. 2001. An interactive effect of simultaneous death of dwarf bamboo, canopy gap, and predatory rodents on beech regeneration. Oecologia 127:281-286.

Agresti, A. 2002. Categorical data analysis. 2a ed. WileyInterscience, New York. 710 p.

Akashi, N. 1997. Dispersion pattern and mortality of seed and seedling of Fagus crenata Blume in a cool temperate forest in Western Japan. Ecological Research 12:159-165.

Alcántara, A. O. y V. I. Luna. 2001. Análisis florístico de dos áreas de bosque mesófilo de montaña en el estado de Hidalgo, México: Eloxochitlán y Tlahuelompa. Acta Botanica Mexicana 54:51-87.

Álvarez-Aquino, C. 1997. Estudio poblacional de Fagus mexicana Martínez en Acatlán, Veracruz. Tesis, Maestría Instituto de Ecología, Xalapa, Veracruz, México. 81 p.

Álvarez-Aquino, C. y G. Williams-Linera. 2002. Seedling bank dynamics of Fagus grandifolia var. mexicana before and after a mast year in a Mexican cloud forest. Journal of Vegetation Science 13:179-184.

Álvarez-Buylla, R. E. y M. Martínez-Ramos. 1990. Seed bank versus seed rain in the regeneration of tropical pioneer tree. Oecologia 84:314-325.

Ángeles-Pérez, G. y M. Sakimoto. 1999. Emergencedisappearance processes and mortality factors of current-year seedlings of Abies firma in a natural Abies-Tsuga forest, Wakayama. Forest Research Kyoto 71:27-33.

Berkowitz, A. R., C. D. Canham y V. R. Kelly. 1995. Competition of tree seedling growth and survival in early successional communities. Ecology 76:11561158.

Brokaw, N. V. L. 1987. Algunos aspectos importantes en 
el estudio de la demografía de plantas en los bosques tropicales. Revista de Biología Tropical 35 (suplemento 1):205-206.

Clark, D. A. y D. B. Clark. 1989. The role of physical damage in the seedling mortality regime of a Neotropical rain forest. Oikos 55:225-230.

Cochran, G. W. 1980. Técnicas de muestreo. CECSA, México, D.F. 513 p.

Collins, S. L. y R. E. Good. 1987. The seedling regeneration niche: habitat structure of tree seedling in an oak-pine forest. Oikos 48:89-98.

Ehnis, D. A. 1981. Fagus mexicana Martínez, su ecología e importancia. Tesis, Facultad de Ciencias, Universidad Autónoma de México, México, D.F. 123 p.

Erickson, O. y J. Ehrlen. 1992. Seed and microsite limitation of recruitment in plant populations. Oecologia 91:360364.

Facelli, J. M. y S. T. A. Pickett. 1991. Indirect effects of litter on woody seedlings subject to herb competition. Oikos 62:129-138.

García, E. 1988. Modificaciones al sistema de clasificación climática de Köppen (para adaptarlo a las condiciones de la República Mexicana). 4a edición. Editado por la autora. México, D.F. 217 p.

Glitzenstein, J. S., P. A Harcombe, y D. R. Streng. 1986. Disturbance, succession, and maintenance of species diversity in an east Texas forest. Ecological Monographs 56:243-258.

Hara, M. 1987. Analysis of seedling bank of a climax beech forest: ecological importance of seedling spouts. Vegetatio 71:67-74.

Harcombe, P. A. 1987. Tree life tables. BioScience 37:557568.

Harper, J. L. 1977. Population biology of plants. Academic, New York. 892 p.

Houle, G. 1992. Spatial relationship between seed and seedling abundance and mortality in a deciduous forest of north-eastern north American. Journal of Ecology 80:99-108.

Ida, H., M. Hottay Y. Ezaki. 2004. Predispersal predation by rodents to beechnuts (Fagus crenata Blume). Ecological Research 19:503-509.

Janzen, D. H. 1971. Seed predation by animals. Annual Review of Ecology and Systematics 2:465-492.

Janzen, D. H. 1978. Seedling patterns of tropical trees. In Tropical trees as living systems, P.B. Tomlinson and M. H. Zimmermann (eds.). Cambridge University, p. 83-128.

Jones, R. H. y R. R. Sharitz. 1989. Potential advantages and disadvantages of germinating early for trees in floodplain forests. Oecologia 81:443-449.

Jones, R. H., B. Allen y R. R. Sharitz. 1997. Why do early- emerging seedlings have survival advantage?: a test using Acer rubrum (Aceraceaae). American Journal of Botany 84:1714-1718.

Jones, R. H., R. R. Sharitz, P. M. Dixon, D. S. Segal y R. L. Schneider. 1994. Woody plant regeneration in four floodplain forests. Ecological Monographs 64:345367.

Lieberman, D. y M. Lieberman. 1987. Forest growth and dynamics at La Selva, Costa Rica (1969-1982). Journal of Tropical Ecology 3, 4 (Suplemento especial):347358.

Luna, V. I. y A. O. Alcántara. 2004. Florística del bosque mesófilo de montaña de Hidalgo. In Biodiversidad de la Sierra Madre Oriental, V. I. Luna, J. J. Morrone y D. Espinosa (eds.). Las prensas de Ciencias, UNAM, México, D.F. p 169-191.

Moles, T. A y M. Westoby. 2004. What do seedling die from and what are the implications for evolution of seed size? Oikos 106:193-199.

Nilsson, G. S. 1985. Ecological and evolutionary interactions between reproduction of beech Fagus sylvatica and seed eating animals. Oikos 44:157-164.

Nilsson, G. S. y U. Wästljung. 1987. Seed predation and cross-pollination in mast-seedling beech (Fagus sylvatica) patches. Ecology 68:260-265.

Pérez-Rodríguez, P. M., 1999. Las hayas de México, monografía de Fagus grandifolia spp. mexicana. Universidad Autónoma Chapingo, Montecillo, Estado de México. 51 p.

Peters, R. 1995. Architecture and development of Mexican beech forest. In Vegetation science in forestry, E.O. Box (ed.) Kluwer Academic, Dordrecht, p. 325-343.

Rowden, A., A. Robertson, T. Allnutt, S. Heredia, G. Williams-Linera. y A. C. Newton. 2004. Conservation generics of Mexican beech, Fagus grandifolia var. mexicana. Conservation Genetics 5:475-484.

SAS Institute 1997. SAS/STAT User Guide, 4a edición, vol. 2. Cary, North Carolina.

Seiwa, K. 1998. Advantages of early germination for growth and survival of seedling of Acer mono under different overstorey phenologies in deciduos broadleaved forest. Journal of Ecology 86:219-228.

Servicio Meteorológico Nacional. 1975. Normales climatológicas. Dirección General de Geografía y Meteorología. Secretaría de Agricultura y Ganadería, México.

Shibata, M. y T. Nakashizuka. 1995. Seed and seedling demography of four co-occurring Carpinus species in a temperate deciduous forest. Ecology 76:099-1108.

Swaine, M. D., D. Lieberman y F. E. Putz. 1987. The dynamics of tree populations in tropical forest: a 
review. Journal of Tropical Ecology 3, 4 (Suplemento especial):359-366.

Szwagrzyk, J., J. Szewezyk y J. Bodziarezyk. 2001. Dynamics of seedling banks in beech forest: results of a 10-year study on germination, growth and survival. Forest Ecology and Management 141:237-250.

Taylor, M. K. y L. W. Aarssen. 1989. Neighbor effects in mast year seedling of Acer saccharum. American Journal of Botany 76:546-554.

Tomita, M., Y. Hirabuki y K. Seiwa. 2002. Post-dispersal changes in the spatial distribution of Fagus crenata seeds. Ecology 83:1560-1565.

Ward, R. T. 1961. Some aspects of regeneration habitats of the American Beech. Ecology 42:828-832.

Williams-Linera, G., A. Rowdeny A. C. Newton. 2003. Distribution and stand characteristics of relict population of Mexican beech (Fagus grandifolia var. mexicana). Biological Conservation 109:27-36.

Yasaka, M., K. Terazawa, H. Koyama y H. Kon. 2003. Masting behavior of Fagus crenata in northern Japan: spatial synchrony and pre-dispersal seed predation. Forest Ecology and Management 184:227-284. 